\title{
Role of SGLT1 in high glucose level-induced $M M P-2$ expression in human cardiac fibroblasts
}

\author{
LIPING MENG ${ }^{1,2}$, HIROYASU UZUI ${ }^{1}$, HANGYUAN GUO $^{2}$ and HIROSHI TADA ${ }^{1}$ \\ ${ }^{1}$ Department of Cardiovascular Medicine, Faculty of Medical Sciences, University of Fukui, Fukui 910-1193, Japan; \\ ${ }^{2}$ Department of Cardiology, Shaoxing People's Hospital, Shaoxing Hospital of Zhejiang University, \\ Shaoxing, Zhejiang 312000, P.R. China
}

Received November 1, 2017; Accepted February 28, 2018

DOI: $10.3892 / \mathrm{mmr} .2018 .8688$

\begin{abstract}
Cardiac fibrosis is a major pathological manifestation of diabetic cardiomyopathy (DCM), which leads to cardiac remodeling, dilated cardiomyopathy and congestive heart failure. Human cardiac fibroblasts (HCF) constitute the predominant cell type in the heart and matrix metalloproteinases (MMPs) and tissue inhibitors of metalloproteinases (TIMPs) are also involved in cardiac fibrosis. However, it is unclear whether high glucose levels affect the expression of MMPs and TIMPs in HCF. Sodium-glucose cotransporter (SGLT) inhibitors have been developed as therapeutic agents and the anti-DCM effect of SGLT inhibitors has been demonstrated by previous studies. However, whether SLGT inhibitors protect the diabetic heart by directly inhibiting the SGLTs in HCF in addition to lowering the blood glucose levels, has not yet been determined. In the present study, increased MMP-2 expression was noted in HCFs in response to high glucose levels, which may be reversed by phlorizin (inhibits both SGLT1 and SGLT2), but not dapagliflozin (inhibits SGLT2). In addition, SGLT1 was revealed to be present in the HCFs and high glucose level was demonstrated to increase SGLT1 expression, which may be attenuated by phlorizin. Therefore it was concluded that high glucose levels induced MMP-2 expression in the HCFs, potentially by upregulating SGLT1. SGLT1 inhibition may be a novel strategy for the treatment of DCM.
\end{abstract}

\section{Introduction}

Diabetes mellitus (DM) is a chronic metabolic disease, affecting many individuals worldwide. The increase in

Correspondence to: Dr Hiroyasu Uzui, Department of Cardiovascular Medicine, Faculty of Medical Sciences, University of Fukui, 23-3 Shimoaizuki Matsuoka Eiheiji-cho, Fukui 910-1193, Japan

E-mail: huzui@u-fukui.ac.jp

Key words: sodium-glucose cotransporter 1, cardiac fibrosis, matrix metalloproteinases, glucose, phlorizin prevalence is followed by a global pandemic of diabetes-related complications. Diabetic cardiomyopathy (DCM) is one of the major complications of DM (1). However, the exact molecular mechanisms underlying DCM remain unclear.

Matrix metalloproteinases (MMPs) are responsible for cleaving extracellular matrix (ECM) proteins. Among the MMPs, MMP-2 and MMP-9 are the major gelatinases that play an important role in the development of DCM by degrading the ECM (2). MMP-2 expression is higher in a diabetic heart $(3,4)$. Moreover, dysregulation of MMP proteins and their endogenous inhibitor, namely, tissue inhibitor of metalloproteinase (TIMP), has been observed in the diabetic heart, suggesting that MMPs/TIMPs are involved in DCM. However, whether high glucose levels affect the expression of MMPs/TIMPs in human cardiac fibroblasts (HCF) is unclear.

Sodium-glucose cotransporters (SGLTs) are encoded by a subfamily of solute carrier genes, which are members of the sodium substrate symporter family. SGLT transport glucose by following the sodium concentration gradient which is established by the $\mathrm{Na}+\mathrm{K}+$-ATPase pump. The primary SGLTs include SGLT1, which accounts for glucose absorption from the small intestine, and SGLT2, which is responsible for reabsorption of the glucose in the proximal renal tubule. SGLT inhibitor has been developed as a novel strategy for the treatment of type 2 DM patients. Recently, the result of EMPA-REG OUTCOME trail demonstrated that, Empagliflozin, a new member of the SGLT2 class, could significantly decreased the cardiovascular morbidity and mortality in DM patients (5). In addition, Cefalu et al (6), showed that dapagliflozin plays a significant role in the reduction of HbA1c, BW, and SBP. However, it had no adverse effect on cardiovascular safety, compared to placebo treatment. In short-term studies, SGLT1 inhibition and combined SGLT1/SGLT2 inhibition were found to be safe (7). SGLT inhibitors play a cardiovascular protective role, possibly by inhibiting renal reabsorption of glucose, thereby lowering blood glucose levels. Recently, SGLT1 was found to be highly expressed in the human and rodent heart, and to actually contribute to the pathogenesis of PRKAG2 cardiomyopathy $(8,9)$. Knockdown of SGLT1 could attenuate the disease phenotype (10). SGLT1 was also found to be expressed in cardiomyocytes (8). However, SGLT expression in the HCF has not been previously tested, and the cardioprotective mechanism of SLGT inhibitors involving direct 
inhibition of the SGLT in HCF, in addition to lowering blood glucose levels, is unclear.

In the present study, we investigated whether high glucose levels regulate the expression of MMPs and TIMPs in HCF. We studied the effect of two SGLT inhibitors (phlorizin and dapagliflozin) on glucose-induced $M M P$-2 expression in the HCF as well as investigated the role of SGLT1 in this effect.

\section{Materials and methods}

Materials. The CS4Z055R (containing serum) and CS4Z3500R (not containing serum) media were purchased from Cell Systems Corporation, (Kirkland, WA, USA). D-(+)-Glucose, D-(+)-mannitol, and diamidino-2-phenylindole (DAPI) were purchased from Nacalai Tesque Inc., (Kyoto, Japan). Phlorizin and dapagliflozin were purchased from Cayman Chemical Company, (Ann Arbor, MI, USA). All other chemicals were of reagent grade and commercially available.

Cell culture. Primary human cardiac fibroblast cells (ACBRI 5118) at passage 2 were purchased from Cell Systems Corporation. All studies were performed with HCFs at passage 4-10. Passage Reagent Group (Cell Systems Corporation) were used for cell passaging. The cells were seeded on 6-well tissue culture plates at a density of $1 \times 10^{5}$ cells/well, maintained in the CS4Z055R medium, and grown in the cell incubator at $37^{\circ} \mathrm{C}$, containing $95 \% \mathrm{O}_{2}$ and $5 \% \mathrm{CO}_{2}$. After sub-confluence, CS4Z3500R (without serum) medium was used to synchronize the cells for $24 \mathrm{~h}$. The cells were washed twice with phosphate-buffered saline and cultured in the medium to be further subjected to different treatments. Cells were passaged in $0.05 \%$ trypsin-EDTA.

Groups and interventions. To test the effect of glucose on HCF, the cells were divided into 7 groups: Control group, Glu $5.5 \mathrm{mM}$ group (cultured with $5.5 \mathrm{mM}$ glucose), Glu $30 \mathrm{mM}$ group (cultured with $30 \mathrm{mM}$ glucose), Glu $100 \mathrm{mM}$ group (cultured with $100 \mathrm{mM}$ glucose), osmotic control (OC) $5.5 \mathrm{mM}$ group (cultured with $5.5 \mathrm{mM}$ mannitol), OC $30 \mathrm{mM}$ group (cultured with $30 \mathrm{mM}$ mannitol), and OC $100 \mathrm{mM}$ group (cultured with $100 \mathrm{mM}$ mannitol). Different times of incubation, including $1,2,4,6,12,24$, and $48 \mathrm{~h}$, were adopted to evaluate the effects of glucose on HCF. Then, to test the effect of phlorizin and dapagliflozin on glucose-induced MMP-2 expression in HCF, the cells were divided into 6 groups: Control group, Glu $30 \mathrm{mM}$ group (cultured with $30 \mathrm{mM}$ glucose), Phlorizin $10 \mu \mathrm{M}$ group (cultured with $30 \mathrm{mM}$ glucose and $10 \mu \mathrm{M}$ phlorizin), Phlorizin $100 \mu \mathrm{M}$ group (cultured with $30 \mathrm{mM}$ glucose and $100 \mu \mathrm{M}$ phlorizin), Dapagliflozin $10 \mu \mathrm{M}$ group (cultured with $30 \mathrm{mM}$ glucose and $10 \mu \mathrm{M}$ dapagliflozin), and Dapagliflozin $100 \mu \mathrm{M}$ group (cultured with $30 \mathrm{mM}$ glucose and $100 \mu \mathrm{M}$ dapagliflozin).

Isolation of total $m R N A$ and reverse transcription-quantitative polymerase chain reaction (RT-qPCR). After group-specific treatment, RNeasy mini-kit (Qiagen GmbH, Hilden, Germany) was used for extracting total RNA from the cells. Then, the mRNA was used as the template to synthesize complementary DNA (cDNA) with Thermo Script RT-PCR kit (Invitrogen; Thermo Fisher Scientific, Inc., Waltham, MA, USA) according to the manufacturer's instructions. Approximately $2 \mu 1$ of the cDNA was used for RT-PCR. The forward and reverse primer sequences are shown in Table I. The reaction conditions included the following: step $1: 95^{\circ} \mathrm{C}$ for $30 \mathrm{sec}$; step 2: 40 cycles at $95^{\circ} \mathrm{C}$ for $5 \mathrm{sec}$ and $60^{\circ} \mathrm{C}$ for $34 \mathrm{sec}$; step $3: 95^{\circ} \mathrm{C}$ for $15 \mathrm{sec}$, $60^{\circ} \mathrm{C}$ for $60 \mathrm{sec}$, and $95^{\circ} \mathrm{C}$ for $15 \mathrm{sec}$. The final concentration of $M M P-2, T I M P-1, T I M P-2$, and SGLT-1 was expressed relative to that of $G A P D H$ from the same RNA sample.

Western blot analysis. After $24 \mathrm{~h}$ incubation with the corresponding intervention factors, the cellular protein was extracted using the radioimmunoprecipitation assay (RIPA) lysis buffer for use in western blotting. This was followed by determination of protein content in the supernatant. The supernatant was then separated by SDS-PAGE (10\%) and transferred to a polyvinylidene fluoride membrane. The membrane was blocked with the blocking buffer for $30 \mathrm{~min}$ at room temperature, and then incubated overnight with the rabbit anti-SGLT1 monoclonal antibody (1:1,000 diluted) and rabbit anti- $\beta$-actin monoclonal antibody $(1: 10,000$ diluted $)$ at $4^{\circ} \mathrm{C}$. After wash with TBS-T for 3 times, the membranes were then incubated with the second antibody (1:10,000 diluted) for $1 \mathrm{~h}$ at room temperature. The antigen was detected by using the standard chemical luminescence method. The bands on the membranes were scanned on a gel imaging system (Bio-Rad Laboratories, Inc., Hercules, CA, USA) and analyzed by Quantity One v4.4.

Statistical analysis. Statistical analysis was performed using the GraphPad Prism (v6.0; GraphPad Software, Inc., La Jolla, CA, USA) software. One-way analysis of variance (ANOVA), followed by Tukey's post-hoc analysis, was performed to compare between multiple experimental groups. $\mathrm{P}<0.05$ was considered to indicate a statistically significant difference.

\section{Results}

High glucose levels induce MMP-2 and TIMP-1 expression in $H C F$. We performed RT-qPCR assay to test the effect of glucose on HCF. As shown in Fig. 1, we found that the relative expression of $M M P-2$ and TIMP-1 was up-regulated by glucose at 30 and $100 \mathrm{mM}(\mathrm{P}<0.05$; Fig. 1A-B). However, high glucose levels did not have any effect on the expression of TIMP-2 (Fig. 1C). As the ratio of MMP-2 to TIMP-2 is usually considered representative of ECM balance $(3,11,12)$, we tested the effect of high glucose levels on it; we found that high glucose levels could increase the MMP-2/TIMP-2 ratio $(\mathrm{P}<0.05$; Fig. 1D). Moreover, MMP-9 mRNA expression was very low, and therefore, we did not test the expression of MMP-9 in the subsequent experiments. We found that mannitol at 30 and $100 \mathrm{mM}$ had no effect on the expression of $M M P-2$ and TIMP-1/2; these results indicated that high glucose levels, and not osmotic effect, were responsible.

Phlorizin inhibits high glucose level-induced MMP-2 and TIMP-1 expression in HCF. Based on the findings of previous studies (13) as well as the above-described results, $30 \mathrm{mM}$ glucose was used to induce the expression of $M M P-2$ and TIMP-1. As shown in Fig. 2, $100 \mu \mathrm{M}$ phlorizin inhibits $M M P-2$ and TIMP-1 expression in HCF, which is induced by high glucose $(\mathrm{P}<0.05)$; however, dapagliflozin had no 
Table I. Reverse transcription-quantitative polymerase chain reaction primer sequences.

\begin{tabular}{|c|c|}
\hline Primer name & Primer sequence $\left(5^{\prime}-3^{\prime}\right)$ \\
\hline MMP-2 (Forward) & CACATCGCAGATGCCTGGAA \\
\hline MMP-2 (Reverse) & TTCAGGTAATAGGCACCCTTGAAG \\
\hline MMP-9 (Forward) & CAAGCTGGACTCGGTCTTTGA \\
\hline MMP-9 (Reverse) & GCCTGTGTACACCCACACCT \\
\hline TIMP-1 (Forward) & AAGAACTACACTGTTGGCTGTGAG \\
\hline TIMP-1 (Reverse) & GTCCGTCCACAAGCAATGAG \\
\hline TIMP-2 (Forward) & GGAGCACTGTGTTTATGCTGGA \\
\hline TIMP-2 (Reverse) & ACATGCGCAGTCTGCTTGTC \\
\hline SGLT-1 (Forward) & GCCCAACACTCTGATTTGCATTTA \\
\hline SGLT-1 (Reverse) & CTGGTTCTACTTCACCCTGAGCAC \\
\hline GAPDH (Forward) & GCACCGTCAAGGCTGAGAAC \\
\hline GAPDH (Reverse) & ATGGTGGTGAAGACGCCAGT \\
\hline
\end{tabular}

MMP, matrix metalloproteinase; TIMP, tissue inhibitor of metalloproteinases; SGLT, sodium-glucose cotransporter.

effect (Fig. 2A-B). Both phlorizin and dapagliflozin had no effect on the expression of TIMP-2 (Fig. 2C). We also found that phlorizin could decrease the ratio of $M M P-2 / T I M P-2$ $(\mathrm{P}<0.05$; Fig. 2D).

Phlorizin inhibits high glucose level-induced SGLT-1 expression in HCF. To determine the involvement of SGLT-1, western blotting was used to detect SGLT-1 expression in each group. As shown in Fig. 3, we found that high glucose levels increase the expression of SGLT-1 in HCF, and phlorizin inhibits the expression of SGLT-1 $(\mathrm{P}<0.05)$. Meanwhile, dapagliflozin did not have this effect. These results indicated that high glucose levels might induce MMP-2 and TIMP-1 expression by up-regulating SGLT-1.

\section{Discussion}

DCM, which was first described in 1972, is defined as myocardial dysfunction in the DM patients without hypertension and coronary artery disease; it could finally result in heart failure (14). There is growing evidence that myocardial fibrosis, cardiomyocyte apoptosis, inflammation, oxidative stress, impaired calcium handling, renin-angiotensin system activation, and mitochondrial dysfunction are involved in the development of DCM (15). Among all these factors, myocardial fibrosis is the most frequently proposed mechanism to explain cardiac changes in DCM. Many studies have shown that inhibiting myocardial fibrosis can attenuate the progress of DCM in animal experiments $(16,17)$.

Cardiac fibroblasts are the main cell type constituting the heart, and are responsible for the basal deposition and degradation of the ECM. As the primary structural cells of the heart, cardiac fibroblasts are critically involved in all cardiac fibrotic conditions. Liu et al (13), showed that high glucose levels induced cardiac fibrosis in diabetic mice by increasing the proliferation of and collagen synthesis by cardiac fibroblasts. In addition, in the in vitro experiment, Wang et al (18), found that high glucose levels induced collagen formation and cytoskeleton degradation in cardiac fibroblasts. Li et al (19), also showed that inhibiting high glucose level-induced proliferation and differentiation of and collagen accumulation by cardiac fibroblasts could be a new therapeutic strategy for diabetes. In this study, we found that high levels of glucose can induce MMP-2 expression in the HCF. MMP-2 is the primary kind of gelatinases that can degrade the ECM. Synthesis and decomposition of ECM are ongoing processes. In the normal heart, the decomposition and synthesis of ECM are in dynamic equilibrium. The balance is maintained by MMPs and TIMPs (20). As TIMP-2 is an important member of the TIMP family, it can effectively inhibit the activity of MMP-2. Therefore, we further determined the MMP-2/TIMP-2 ratio and found that high glucose level also increases this ratio. Thus, these results indicate that high glucose levels can degrade the ECM and attenuate cardiac fibrosis by up-regulating $M M P-2$. However, as we know, during cardiac remodeling, ECM degradation and synthesis are activated simultaneously. Derangement of $M M P-2$ expression and activity alters the balance between ECM synthesis and degradation, resulting in excessive collagen deposition and reduced structural integrity in the myocardium. Increasing degradation of ECM supplies space for the proliferation and migration of HCF and other macrophagocytes, which secrete inflammatory and growth factors, further enhancing the cardiac remodeling process (21). Siddesha et al (22), showed that sustained induction and activation of MMPs and the destruction and deposition of ECM can result in cardiac fibrosis. In addition to its canonical function in ECM degradation, studies in the recent year have highlighted new functions of MMP-2 to induce cardiac conditions such as proteolysis of novel substrates other than ECM proteins such as troponin I (23), localization to subcellular organelles like the mitochondria (24), and proteolysis of susceptible intracellular proteins in subcellular compartments, such as monocyte chemoattractant protein-3 (25). All these functions subsequently resulted in cardiac remodeling and heart failure. In accordance with our results, animal studies also showed that MMP-2 expression and activity was increased in the diabetic heart $(3,4)$. Therefore, we suggest that high glucose levels up-regulate the expression of MMP-2, which further promotes ECM degradation, increased HCF migration and proliferation, finally resulting in fibrosis and DCM.

SGLT1 has been reported to exist in the small intestine, skeletal muscle, heart, kidney, trachea, prostate, cervix, and mesenteric adipose tissue (26). It is also expressed in the kidney and intestine (26). In the heart, SGLT1 has been shown to be expressed in the cardiomyocytes and endothelial cells (27). In the present study, we found that SGLT1 was expressed in HCF. This is, to the best of our knowledge, the first report that SGLT1 is present in HCF. In addition, we found that SGLT1 is up-regulated by glucose levels. Previous studies had found that SGLT1 is substantially expressed in the myocardium and actually contributes to the pathogenesis of PRKAG2 cardiomyopathy $(8,9)$, and that knockdown of SGLT1 can attenuate the disease phenotype (10). Thus, SGLT1 might be involved in DCM. In order to explore the effect of SGLT1, we used phlorizin (inhibits SGLT1 and SGLT2) and dapagliflozin (inhibits SGLT2). Our results showed that phlorizin can inhibit SGLT-1 expression in HCF. In addition, phlorizin can inhibit 
A

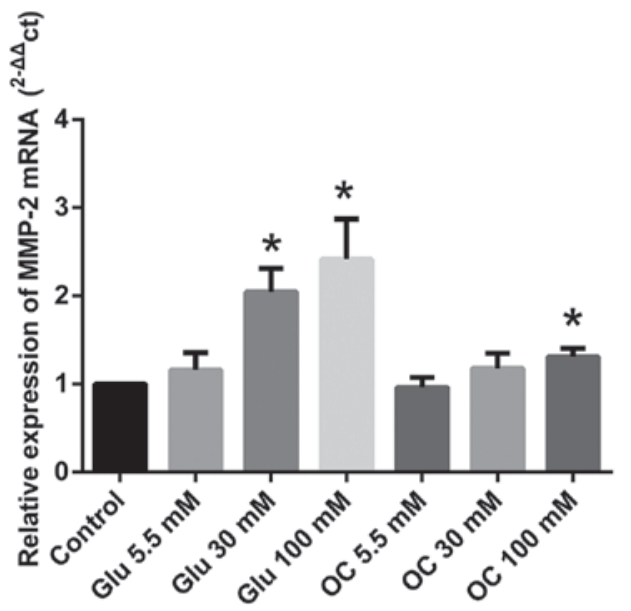

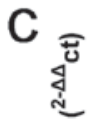

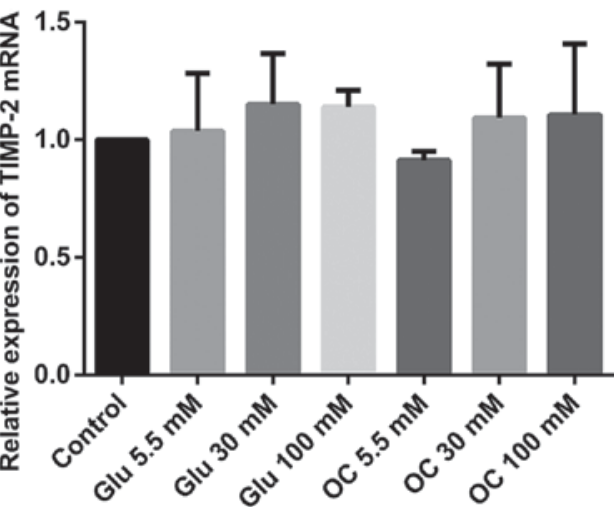

B

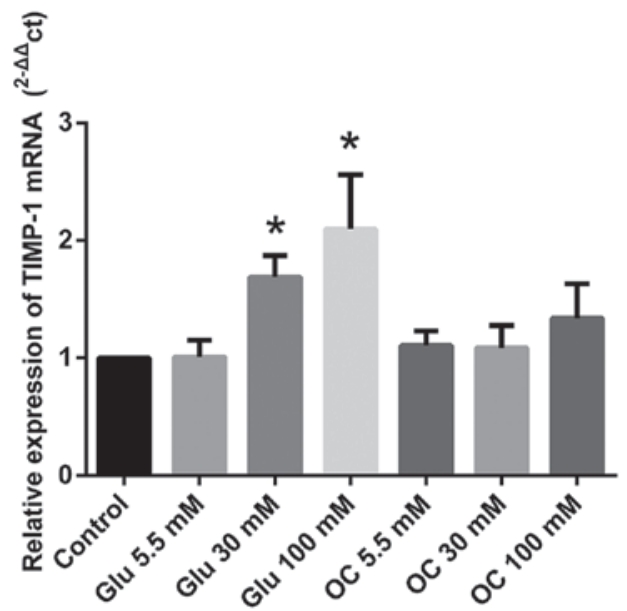

D

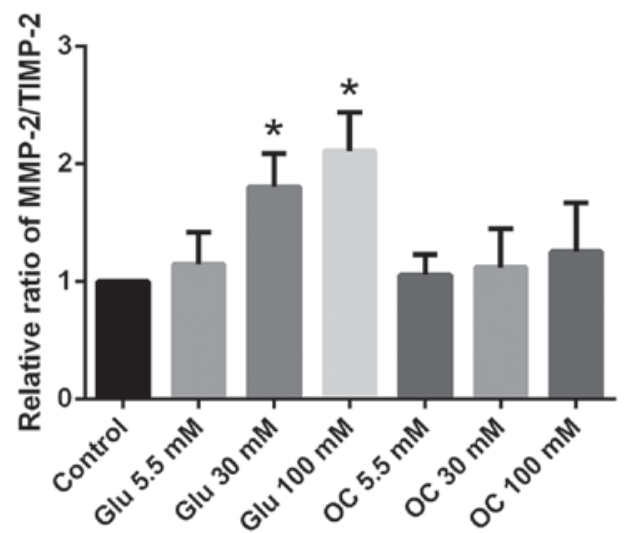

Figure 1. High levels of glucose induce $M M P-2$ and TIMP-1 expression in HCF. Relative expression of (A) MMP-2, (B) TIMP-1 and (C) TIMP-2 mRNA in each group. (D) Relative ratio of $M M P-2 / T I M P-2$ in each group. ${ }^{*} \mathrm{P}<0.05$ vs. the control. MMP, matrix metalloproteinase; TIMP, tissue inhibitor of metalloproteinases.

high glucose level-induced MMP-2 and TIMP-1 expression in HCF. Also, dapagliflozin did not exert this effect. These results indicated that the up-regulation of SGLT1 is necessary for the induction of MMP-2 expression by high glucose levels, and that the inhibition of SGLT1 can attenuate this effect. Balteau et al (28), showed that SGLT1 is linked with NADPH oxidase activation. Later, Van Steenbergen et al (29), found that SGLT1 mediated the production of reactive oxygen species induced by hyperglycemia in the heart. Both NADPH oxidase activation and production of reactive oxygen species enhanced MMP-2 expression and activation. Thus, this might be the mechanism involved in the down-regulation of MMP-2 expression by inhibited SGLT1. In the subsequent experiment, we will use siRNA to knock down SGLT1 and over express SGLT1 to further verify the relationship between SGLT1 and MMP-2.

In a recent study, myocardial ischemia and hypertrophy were found to be associated with SGLT1 up-regulation, while SGLT2 was not expressed (30). SGLT1 inhibition in the heart, which was previously thought to inhibit SGLT1 expression in the cardiomyocytes, could result in potential improvement of cardiac function and reduction of arrhythmic risk. Our study suggests another mechanism used by SGLT1 to protect the diabetic heart, that is, by attenuating glucose-induced $M M P-2$ expression in HCF. Although dapagliflozin has demonstrated anti-DCM effect in previous studies $(31,32)$, in the present study, it showed no effect on glucose-induced MMP2 expression. The possible mechanism can be down-regulation of serum glucose levels or direct influence on the cardiac tissue through some unknown mechanism.

In summary, inhibition of MMP-2 expression is suggested to be cardioprotective in diabetes. In this study, we showed that $M M P-2$ expression increased in the $\mathrm{HCF}$ in response to high glucose levels, which could be reversed by phlorizin, but not by dapagliflozin. In addition, we found that SGLT1 exists in the HCF and that high glucose levels increase the expression of SGLT1 in HCF, which could also be attenuated by phlorizin. Thus, we concluded that high glucose levels induce $M M P-2$ expression in HCF, possibly by up-regulation of SGLT1.

This study has some limitations. First, we only tested the mRNA levels of MMP-2, MMP-9, TIMP-1, and TIMP-2; we have not evaluated the expression of these proteins by western blotting. Second, SGLT1 over-expression or knock-down has not been used to further verify the relationship between SGLT1 and MMP-2. Third, it is only an in vitro experiment using one cell line; evaluation of other cell lines and in vivo 

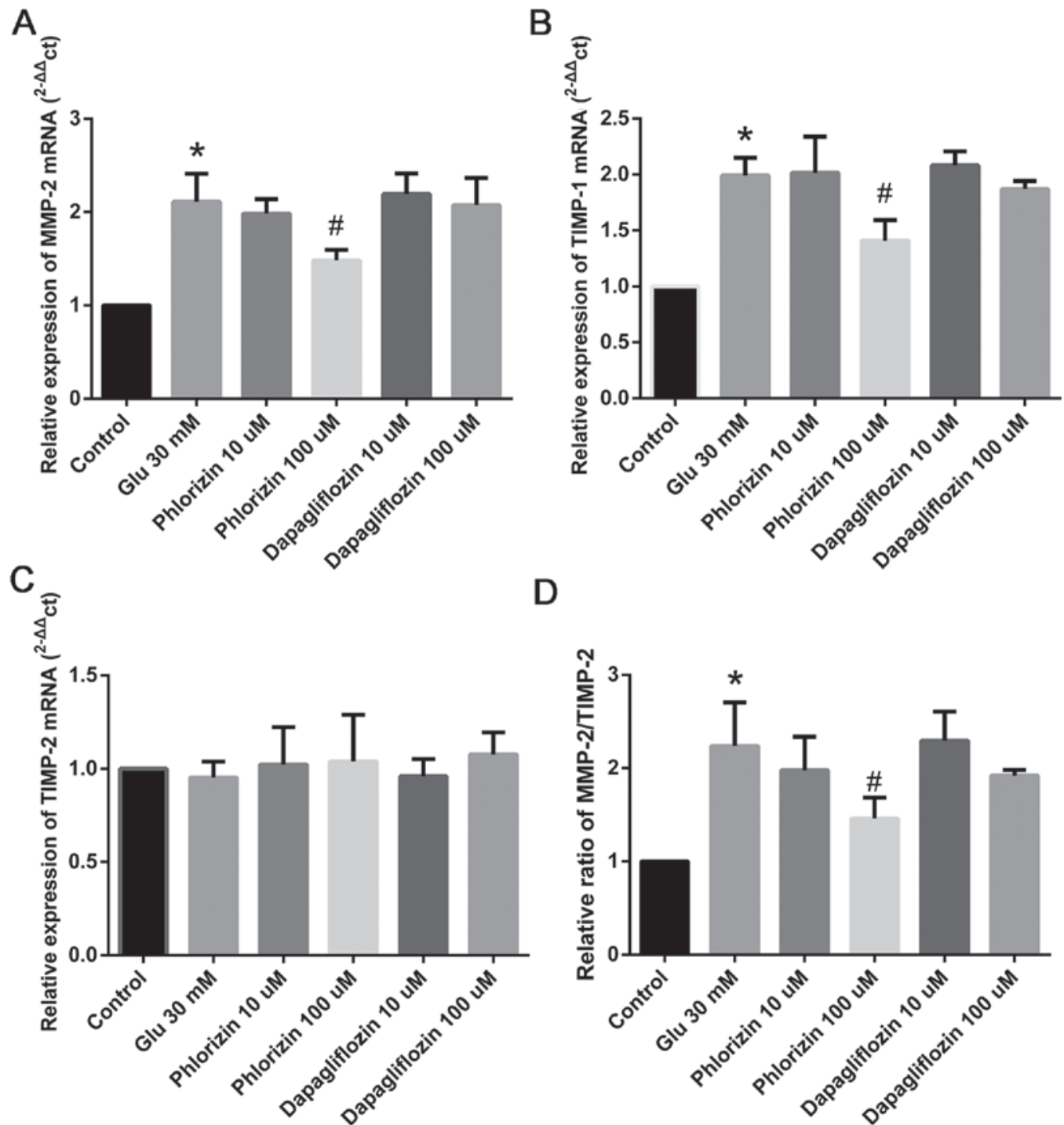

Figure 2. Phlorizin inhibits high glucose level-induced MMP-2 and TIMP-1 expression in HCF. Relative expression of (A) $M M P-2$, (B) TIMP-1 and (C) TIMP-2 mRNA in each group. (D) Relative ratio of MMP-2/TIMP-2 in each group. ${ }^{*} \mathrm{P}<0.05$ vs. the control; " $\mathrm{P}<0.05$ vs. the Glu $30 \mathrm{mM}$ group. MMP, matrix metalloproteinase; TIMP, tissue inhibitor of metalloproteinases.
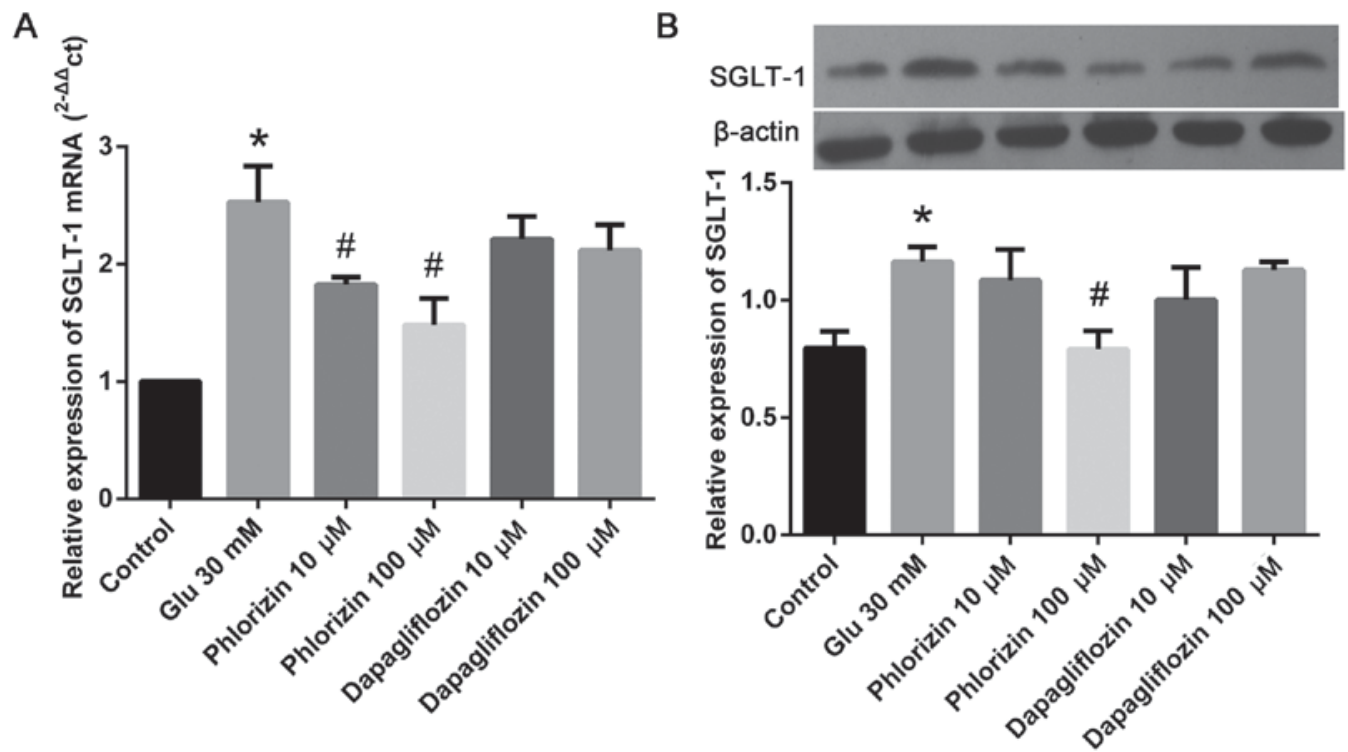

Figure 3. Phlorizin inhibits high glucose level-induced SGLT-1 expression in HCF.Relative expression of SGLT-1 (A) mRNA and (B) protein in each group. $\mathrm{P}<0.05$ vs. the control; " $\mathrm{P}<0.05$ vs. the Glu $30 \mathrm{mM}$ group. MMP, matrix metalloproteinase; TIMP, tissue inhibitor of metalloproteinases. 
experiments should be conducted in future to verify this conclusion.

\section{Acknowledgements}

The present study was supported by the Basic Public Welfare Research Project of Zhejiang Province (LGF18H020009) and the Youth Research Fund Project of Shaoxing People's Hospital (2017A02).

\section{References}

1. Fang ZY, Prins JB and Marwick TH: Diabetic cardiomyopathy: Evidence, mechanisms and therapeutic implications. Endocr Rev 25: 543-567, 2004.

2. DeCoux A, Lindsey ML, Villarreal F, Garcia RA and Schulz R: Myocardial matrix metalloproteinase-2: Inside out and upside down. J Mol Cell Cardiol 77: 64-72, 2014.

3. Li Q, Sun SZ, Wang Y, Tian YJ and Liu MH: The roles of MMP-2/TIMP-2 in extracellular matrix remodelling in the hearts of STZ-induced diabetic rats. Acta Cardiol 62: 485-491, 2007.

4. Chen SL, Hu ZY, Zuo GF, Li MH and Li B: I(f) current channel inhibitor (ivabradine) deserves cardioprotective effect via down-regulating the expression of matrix metalloproteinase (MMP)-2 and attenuating apoptosis in diabetic mice. BMC Cardiovasc Disord 14: 150, 2014

5. Zinman B, Wanner C, Lachin JM, Fitchett D, Bluhmki E, Hantel S, Mattheus M, Devins T, Johansen OE, Woerle HJ, et al: Empagliflozin, cardiovascular outcomes and mortality in type 2 diabetes. N Engl J Med 373: 2117-2128, 2015.

6. Cefalu WT, Leiter LA, de Bruin TW, Gause-Nilsson I, Sugg J and Parikh SJ: Dapagliflozin's effects on glycemia and cardiovascular risk factors in high-risk patients with type 2 diabetes: A 24 -week, multicenter, randomized, double-blind, placebo-controlled study with a 28-week extension. Diabetes Care 38: 1218-1227, 2015.

7. Song P, Onishi A, Koepsell H and Vallon V: Sodium glucose cotransporter SGLT1 as a therapeutic target in diabetes mellitus. Exp Opin Ther Targets 20: 1109-1125, 2016.

8. Kashiwagi Y, Nagoshi T, Yoshino T, Tanaka TD, Ito K, Harada T, Takahashi H, Ikegami M, Anzawa R and Yoshimura M: Expression of SGLT1 in human hearts and impairment of cardiac glucose uptake by phlorizin during ischemia-reperfusion injury in mice. PLoS One 10: e0130605, 2015.

9. Banerjee SK, Ramani R, Saba S, Rager J, Tian R, Mathier MA and Ahmad F: A PRKAG2 mutation causes biphasic changes in myocardial AMPK activity and does not protect against ischemia. Biochem Biophys Res Commun 360: 381-387, 2007.

10. Ramratnam M, Sharma RK, D'Auria S, Lee SJ, Wang D, Huang XY and Ahmad F: Transgenic knockdown of cardiac sodium/glucose cotransporter 1 (SGLT1) attenuates PRKAG2 cardiomyopathy, whereas transgenic overexpression of cardiac SGLT1 causes pathologic hypertrophy and dysfunction in mice. J Am Heart Assoc 3: e000899, 2014.

11. Das S, Mandal M, Chakraborti T, Mandal A and Chakraborti S: Isolation of MMP-2 from MMP-2/TIMP-2 complex: Characterization of the complex and the free enzyme in pulmonary vascular smooth muscle plasma membrane. Biochim Biophys Acta 1674: 158-174, 2004

12. Avolio C, Filippi M, Tortorella C, Rocca MA, Ruggieri M, Agosta F, Tomassini V, Pozzilli C, Stecchi S, Giaquinto P, et al: Serum MMP-9/TIMP-1 and MMP-2/TIMP-2 ratios in multiple sclerosis: Relationships with different magnetic resonance imaging measures of disease activity during IFN-beta-1a treatment. Mult Scler 11: 441-446, 2005.

13. Liu X, Song X, Lu J, Chen X, Liang E, Liu X, Zhang M, Zhang Y, Du Z and Zhao Y: Neferine inhibits proliferation and collagen synthesis induced by high glucose in cardiac fibroblasts and reduces cardiac fibrosis in diabetic mice. Oncotarget 7: 61703-61715, 2016.

14. Rubler S, Dlugash J, Yuceoglu YZ, Kumral T, Branwood AW and Grishman A: New type of cardiomyopathy associated with diabetic glomerulosclerosis. Am J Cardiol 30: 595-602, 1972.

15. Jia G, Whaley-Connell A and Sowers JR: Diabetic cardiomyopathy: A hyperglycaemia- and insulin-resistance-induced heart disease. Diabetologia 61: 21-28, 2017.
16. Zou C, Liu X, Xie R, Bao Y, Jin Q, Jia X, Li L and Liu R: Deferiprone attenuates inflammation and myocardial fibrosis in diabetic cardiomyopathy rats. Biochem Biophys Res Commun 486: 930-936, 2017.

17. Lo SH, Hsu CT, Niu HS, Niu CS, Cheng JT and Chen ZC: Cryptotanshinone inhibits STAT3 signaling to alleviate cardiac fibrosis in type 1-like diabetic rats. Phytother Res 31: 638-646, 2017.

18. Wang XW, Zhang FX, Yang F, Ding ZF, Agarwal N, Guo ZK and Mehta JL: Effects of linagliptin and liraglutide on glucoseand angiotensin II-induced collagen formation and cytoskeleton degradation in cardiac fibroblasts in vitro. Acta Pharmacol Sin 37: 1349-1358, 2016.

19. Li J, Dai Y, Su Z and Wei G: MicroRNA-9 inhibits high glucose-induced proliferation, differentiation and collagen accumulation of cardiac fibroblasts by down-regulation of TGFBR2. Biosci Rep 36: e00417, 2016.

20. Meng L, Liu L, Zhou C, Pan S, Zhai X, Jiang C, Guo Y, Ji Z, Chi J, Peng F and Guo H: Polyphenols and polypeptides in chinese rice wine inhibit homocysteine-induced proliferation and migration of vascular smooth muscle cells. J Cardiovasc Pharmacol 67: 482-490, 2016.

21. Shi YF, Chi JF, Tang WL, Xu FK, Liu LB, Ji Z, Lv HT and Guo HY: Effects of rosuvastatin on the production and activation of matrix metalloproteinase-2 and migration of cultured rat vascular smooth muscle cells induced by homocysteine. J Zhejiang Univ Sci B 14: 696-704, 2013.

22. Siddesha JM, Valente AJ, Sakamuri SS, Yoshida T, Gardner JD, Somanna N, Takahashi C, Noda M and Chandrasekar B: Angiotensin II stimulates cardiac fibroblast migration via the differential regulation of matrixins and RECK. J Mol Cell Cardiol 65: 9-18, 2013.

23. Cauwe B and Opdenakker G: Intracellular substrate cleavage: A novel dimension in the biochemistry, biology and pathology of matrix metalloproteinases. Crit Rev Biochem Mol Biol 45: 351-423, 2010.

24. Hughes BG, Fan X, Cho WJ and Schulz R: MMP-2 is localized to the mitochondria-associated membrane of the heart. Am J Physiol Heart Circ Physiol 306: H764-H770, 2014.

25. Westermann D, Savvatis K, Lindner D, Zietsch C, Becher PM, Hammer E, Heimesaat MM, Bereswill S, Volker U, Escher F, et al: Reduced degradation of the chemokine MCP-3 by matrix metalloproteinase- 2 exacerbates myocardial inflammation in experimental viral cardiomyopathy. Circulation 124: 2082-2093, 2011.

26. Chen J, Williams S, Ho S, Loraine H, Hagan D, Whaley JM and Feder JN: Quantitative PCR tissue expression profiling of the human SGLT2 gene and related family members. Diabetes Ther 1: 57-92, 2010.

27. Jin X, Yi L, Chen ML, Chen CY, Chang H, Zhang T, Wang L, Zhu JD, Zhang QY and Mi MT: Delphinidin-3-glucoside protects against oxidized low-density lipoprotein-induced mitochondrial dysfunction in vascular endothelial cells via the sodium-dependent glucose transporter SGLT1. PLoS One 8: e68617, 2013.

28. Balteau M, Tajeddine N, de Meester C, Ginion A, Des Rosiers C, Brady NR, Sommereyns C, Horman S, Vanoverschelde JL, Gailly P, et al: NADPH oxidase activation by hyperglycaemia in cardiomyocytes is independent of glucose metabolism but requires SGLT1. Cardiovasc Res 92: 237-246, 2011.

29. Van Steenbergen A, Balteau M, Ginion A, Ferte L, Battault S, Ravenstein CM, Balligand JL, Daskalopoulos EP, Gilon P, Despa F, et al: Sodium-myoinositol cotransporter-1, SMIT1, mediates the production of reactive oxygen species induced by hyperglycemia in the heart. Sci Rep 7: 41166, 2017.

30. Di Franco A, Cantini G, Tani A, Coppini R, Zecchi-Orlandini S, Raimondi L, Luconi M and Mannucci E: Sodium-dependent glucose transporters (SGLT) in human ischemic heart: A new potential pharmacological target. Int J Cardiol 243: 86-90, 2017.

31. Ye Y, Bajaj M, Yang HC, Perez-Polo JR and Birnbaum Y: SGLT-2 inhibition with dapagliflozin reduces the activation of the Nlrp3/ASC inflammasome and attenuates the development of diabetic cardiomyopathy in mice with type 2 diabetes. Further augmentation of the effects with saxagliptin, a DPP4 inhibitor. Cardiovasc Drugs Ther 31: 119-132, 2017.

32. Joubert M, Jagu B, Montaigne D, Marechal X, Tesse A, Ayer A, Dollet L, Le May C, Toumaniantz G, Manrique A, et al: The sodium-glucose cotransporter 2 inhibitor dapagliflozin prevents cardiomyopathy in a diabetic lipodystrophic mouse model. Diabetes 66: 1030-1040, 2017. 